\author{
TOMASZ PRzedZIŃski \\ PABlo Roig \\ Olga Shekhovtsova \\ ZBIGNIEW WĄS \\ JAKUB ZAREMBA

\section{CONFRONTING THEORETICAL} \\ PREDICTIONS WITH EXPERIMENTAL DATA; \\ A FITTING STRATEGY \\ FOR MULTI-DIMENSIONAL DISTRIBUTIONS
}

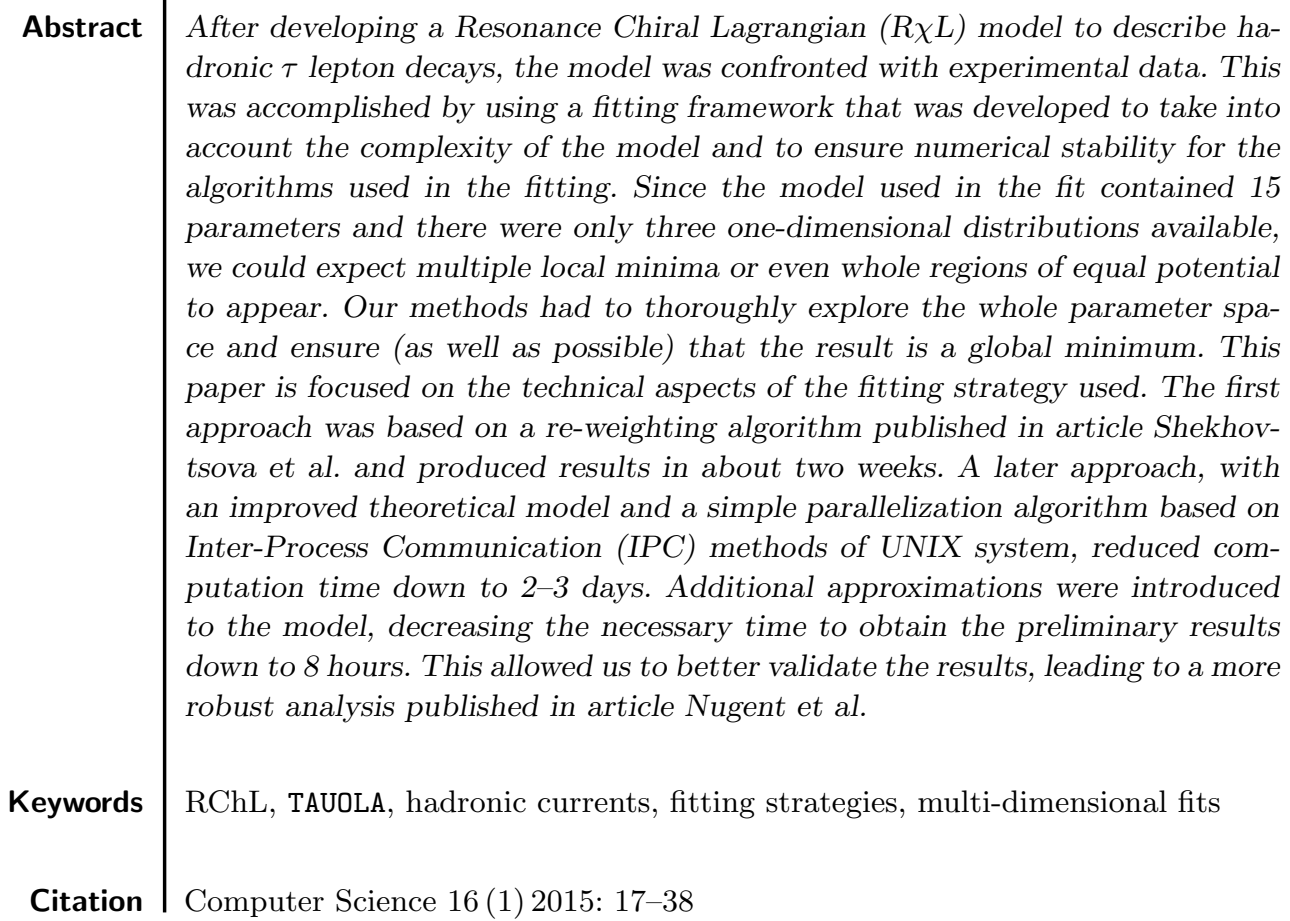




\section{Introduction}

Comparison of the theoretical predictions with experimental data for $\tau$ lepton decays requires sophisticated development strategies that are able to deal with unique problems uncovered during the development process. On the one hand, due to the nature of $\tau$ lepton decays, different decay modes can be analyzed separately to limit the complexity of the project. On the other hand, due to significant cross-contamination between these decay modes, more than one channel needs to be analyzed simultaneously, starting from a certain precision level. A change of parameterization of one can significantly influence the results of the fits of another, as it contributes to its background. New algorithms and new fitting strategies must be designed in a flexible, modular way to account for a variety of changes and extensions that can be introduced throughout the evolution of the project. This is, however, a daunting task, as the nature of the new extensions to the theoretical models as well as the format or applicability of future data are hard to predict. It is often necessary to design more than one approach to the same problem to cover a wide variety of potential use cases.

The new tools developed for such projects must be validated and constantly tested with every change. This requires an extensive test environment developed along with the new tools. When put together with the development of the distributed computing framework necessary for efficient calculations, such a project proves to be a challenging task.

In this paper, we will focus on the computing side of a project aimed at comparing the theoretical predictions for $\tau$ lepton decays with the data. Design and development of refined data analysis is an essential element of an effort necessary for breakthrough discoveries, such as the recent discovery of the Higgs boson. A well-validated and widely-applicable fitting strategy can serve as a basis for future projects of a similar nature, as it often took place in the past (see e.g., [18]).

Let us stress that the development of the model and discussion of the experimental data including systematic errors used in our work is by far beyond the scope of the present publication, where we concentrate on the computing aspects of the work only. For the other aspects and physics details, we address the reader to references $[16,10]$ as well as the references herein, for presentation of this rich and complicated activity.

\subsection{The modeled process}

The data used in this work was collected at a high-energy physics experiment, the BaBar Experiment [4] located at the Stanford Linear Accelerator Center (SLAC). At this experiment, electrons and anti-electrons (positrons) are collided to create new particles of higher energy, which subsequently decay. A long series of such collision events are collected. The decay products resulting from these collisions can be analyzed from the measurement of the sub-atomic particles produced in the decay as they traverse the detectors that measure the energy, momentum, and velocity of the par- 
ticles. The target of our analysis is to compare the data measured by the experiment to predictions provided by theoretical models.

Our analysis focuses on the decay of the $\tau$ lepton. The $\tau$ lepton is the heaviest of the three known charged leptons and is the only one that decays into pions and kaons. An example of lepton production and decay is presented in Figure 1.

The $\tau$ lepton can decay into multiple channels; thus, we narrow down our focus by analyzing only one possible decay channel. We have chosen the most significant nontrivial decay channel, which is the decay to three charged pions $\left(\tau^{ \pm} \rightarrow \pi^{ \pm} \pi^{ \pm} \pi^{\mp} \nu_{\tau}\right)$ mediated mainly by the axial vector $a_{1}^{ \pm}$(1260) resonance [12]. The experimental data describing the result of such a process consist of a set of distributions of the invariant masses of the decay products.

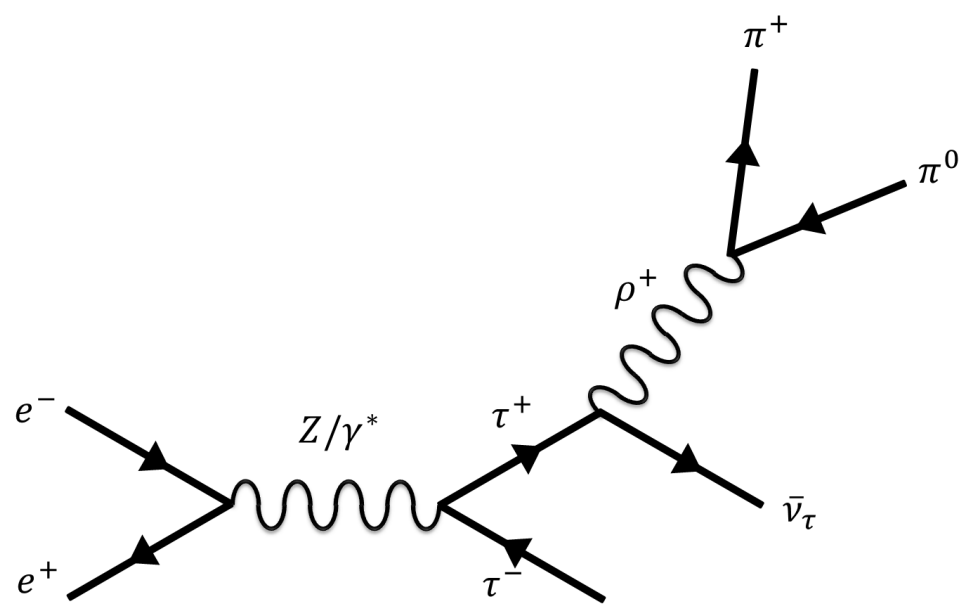

Figure 1. An example of a $e^{+} e^{-} \rightarrow Z / \gamma^{*} \rightarrow \tau^{+} \tau^{-}, \tau^{+} \rightarrow \rho^{+} \rightarrow \pi^{+} \pi^{0} \bar{\nu}_{\tau}$ decay chain. One of the $\tau$ leptons decays to a pair of $\pi$ through the resonance $\rho$. The $\tau$ neutrino $\left(\nu_{\tau}\right)$ escapes detection. The decay of the second $\tau$ is omitted for simplicity.

In the case of a $\tau^{-}$decay, these distributions are: $\pi^{-} \pi^{-}, \pi^{-} \pi^{+}, \pi^{-} \pi^{+}$and $\pi^{-} \pi^{-} \pi^{+}$invariant masses ${ }^{1}$. The charge is inverted for the $\tau^{+}$decays. Because the $\pi^{-}$particles are identical and indistinguishable, the experimental analysis combined the two $\pi^{-} \pi^{+}$pairs into one distribution. The use of one-dimensional distributions by construction neglects correlations between the invariant mass distributions and is not optimal. However, it was decided to use the data currently available from the BaBar experiment [11] to develop the techniques and methods required to address the problems of this fit and to start the collaboration between theorists and experimentalists on this topic.

\footnotetext{
${ }^{1} \mathrm{~A}$ numerical integration over two dimensions is needed to obtain one-dimensional distribution of the three-(two-)pion system invariant mass.
} 
In this paper, we will concentrate on the computing aspects of the work for phenomenology of $\tau \rightarrow \pi \pi \pi \nu_{\tau}$ decays already documented in Refs. [16, 15, 10], where results of the measurements presented in [11] were used.

\subsection{Motivation}

After decades of research by many theorists, the construction of a quantum effective field theory driven by QCD in the energy region populated by resonances ( 1 to $2 \mathrm{GeV}$ ) is still an unsolved problem. However, its low- and high-energy limits are known, which turns out to be extremely useful information to constrain resonance Lagrangians. Tau decays provide us with an opportunity to study low energy QCD interactions and the hadronic currents near and below the perturbative threshold. Unlike the $e^{+} e^{-} \rightarrow$ hadrons decays, the $\tau$ 's decay weakly and, thus, provide access to both the vector and axial vector currents. With the large statistics from the BaBar collaboration, there is the potential to improve the knowledge gained from previous experiments like CLEO [9]. For this purpose, a framework for validating new models must be introduced and a collaboration between theoreticians and experimentalists must be established. Comparing predictions of several different models with the data will help us determine which assumptions are most important in the studies of hadronic currents.

\subsection{Description of the problem}

On the theoretical side, we have a Monte Carlo (MC) simulation aimed at modeling the whole decay chain structure. The purpose of this simulation is to describe the data using a model built on the best knowledge about the decay process.

The first problem that immediately becomes obvious is the fact that the data consist of three one-dimensional distributions while the model has $7-15$ parameters, depending on the effects taken into account, and describes an eight-dimensional space. For such a functional form, the fit (in this case a $\chi^{2}$ ) can be expected to have multiple minima or even whole regions of equal potential. Therefore, we must be able to verify that our methodology is correct and the result is, in fact, a global minimum.

Lastly, we have to keep in mind the time of computation. MC simulations require lengthy calculations for a single generated sample. This makes it impossible to thoroughly scan the whole parameter space to make sure that the result of our fits is a global minimum. While testing and working on improving the theoretical model, we also have to work on the computing strategy.

In following two sections, we will present two approaches to this task: a gradient method for a template morphing fit and a method based on semi-analytical computations. The template morphing technique, where the MC events are re-weighted based on the changes of theoretical parameterization, has several distinct advantages. Firstly, it allows one to correctly incorporate the efficiency and resolution of the detector into a higher-order dimensional fit independently of the observables used in the fit 
and the functional form of the efficiency and resolution. In contrast, standard techniques such as parameterization of the efficiency and resolution (typically by using projections onto a small number of dimensions) have problems when the efficiency or resolution depends strongly on the location in the multi-dimensional space. Moreover, in the case of parameterizations using projections, correlations between the efficiency and resolution are neglected, and regions with extremely high statistics for the model used for the parameterization can bias the parameterization of the efficiency and resolution. The template-morphing technique developed here solves all of these problems. Secondly, it allows for multiple channels that may have different functional dependencies on the fit parameters and/or efficiency and resolution to be easily included in the same fit. The second method, which is based on semi-analytical computations, is only possible due to the experimentalists providing unfolding detector effects. However, this is not always possible.

\subsection{State of art}

For multi-dimensional fits, we decided to use the MINUIT algorithm [8], the primary fitting package used in experimental particle physics, which is available through the ROOT framework [2]. The MINUIT package was developed in 1970s to provide several different approaches to function minimization, including several tools for error estimation and parameter-correlation calculation. During more than four decades of its existence, this package has been extensively used in particle physics. Use of MINUIT is not straightforward and requires understanding of its functionality and proper interpretation of its results. However, it does provide a wide range of control over the fitting procedure.

In terms of a physics simulation, in the case of $\tau$ lepton decays, the leading MC generator TAUOLA [7] was created in 1990 and was based on the model refined later by CLEO collaboration (see e.g. [3]). Since then, it was updated with a new parameterization and was augmented further to take into account new effects discovered over the years. However, even with these improvements, the model is far from perfect, prompting a search for a better solution. The experimental community has made many refinements to the current set of models in TAUOLA for efficiency studies, based mainly on empirical observation without theoretical input; however, most of them remain private.

Non-perturbative QCD is a rather complicated subject. However, its low- and high-energy limits are known. In our context, the latter amounts to use form factors that vanish at infinity. For the former, the CLEO model uses the lowest-order approximation. An alternative theoretical model, Resonance Chiral Lagrangian $(\mathrm{R} \chi \mathrm{L})[6]$, is based on Chiral Perturbation Theory and reproduces its results, at least up to next-to-leading order corrections. The interpolation between the two known extreme limits should be more reliable for the $\mathrm{R} \chi \mathrm{L}$ model, which is why we decided to use it in our analysis. It should be noted here that one of the observations by CLEO was 
that including all of the observed resonances was essential for agreement between data and $\mathrm{MC}^{2}$.

\subsection{Scope}

The goal of this analysis is to develop and test strategies for fitting models to the experimental data and to identify potential problems. These strategies will then be used to test $\chi$ to describe hadronic $\tau$ lepton decays. This means fitting a set of parameters, within their ranges given by the theoretical model, to the experimental data. The result of these fits will show the potential of the new model to represent the data. At the same time, the performance of the fitting strategy can be evaluated, which will be beneficial for future applications.

As it will be shown later, our goal is not only to obtain numerical results from the model as precisely as possible, but also to ensure that our estimation of the firstand second-order derivatives are numerically stable in respect to changes of model parameters. This is a non-trivial issue and provides constraints on our numerical methods. It also affects computing time, sometimes in a critical way, both when searching for the minimum and in the estimation of systematic errors for the fit parameters. Having two different methods helps to ensure the validity of the results and creates a more-robust fitting framework.

In the next section, we describe our first approach to the problem based on the re-weighting algorithm used to compare different parameterizations of a model or different models of $\tau$ decays by applying weights to a data sample generated using the Monte-Carlo simulation [1]. Section 3 describes the second approach based on semi-analytic distributions along with improvements introduced to the physics model and fitting framework. It also introduces the new parallelization algorithm based on Inter-Process Communication (IPC) methods from UNIX systems. The results of the fits, as well as the results of the additional tests performed to validate them, are gathered in Section 4. Summary, Section 5, closes this paper.

\section{Monte-Carlo supported gradient method}

\subsection{Re-weighting algorithm}

When constructing a fit that uses a template constructed from the MC simulation, an important problem is the statistical fluctuations of the sample produced. To be more explicit, MC samples are generated using random numbers; therefore, the accuracy of the predictions is limited by the statistical fluctuations, and therefore, two MC distributions generated with the same parameters will differ due to statistical fluctuations.

\footnotetext{
${ }^{2}$ In the description of hadronic currents predicted by $\mathrm{R} \chi \mathrm{L}$, the low energy large Nc expansion of QCD $[17,19]$ results are represented by amplitudes featuring, in principle, an infinite number of resonances. In practice, their number and parameters are obtained from the fits to the results of the measurements. In our case, the following resonances were necessary: $\rho, \rho^{\prime}, a_{1}, \sigma$, listed in [12], which lead to the enhancement of two-scalar and/or three-scalar propagators.
} 
This is an obstacle for the fitting procedure, which is sensitive to small fluctuations in the predictions. To solve this, a re-weighting algorithm [1], outlined in Figure 2, has been prepared (see [5] for the recent example of its use).

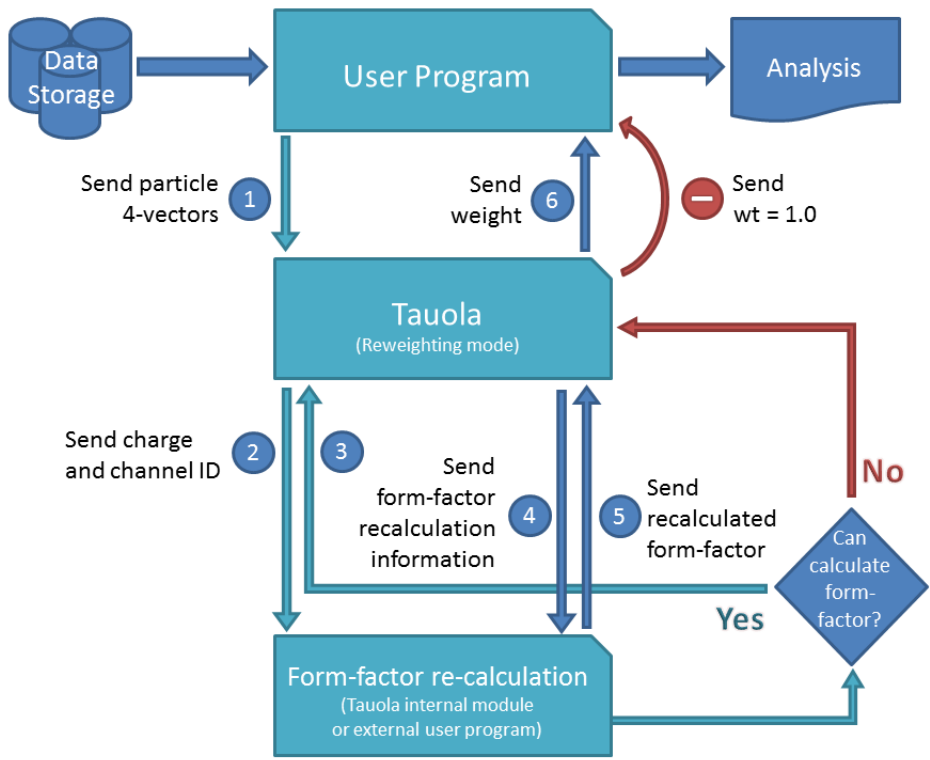

Figure 2. Main concept of the re-weighting algorithm.

For the single generated MC sample, one can calculate weights signifying the change of the matrix element between two theoretical models. The matrix element squared is obtained for both models and then the weight is calculated as their ratio (for example, the $\chi$ model and the CLEO parameterization or two different parameterizations of the $\mathrm{R} \chi \mathrm{L}$ model). For a given sample, one weight per event is calculated, creating a vector of weights that can be used in the fits. This approach eliminates the need for a lengthy MC simulation; instead, attributing weights to a previouslygenerated data sample ${ }^{3}$.

It is worth noting that this approach can be used on a data sample even after all detector and experimental acceptance effects are taken into account. This is a significant factor, considering the computing power needed to perform the detector simulation. Moreover, re-weighting a sample takes significantly less time than generating a new sample with the same number of events, increasing the computation time benefit of this approach.

\footnotetext{
${ }^{3}$ It is essential that we re-use the same sample of events. Thanks to this, statistical fluctuation of the samples does not affect the functional derivatives of the distributions, but affect the shape of the differences only.
} 


\subsection{Approximating the model}

Despite all of its benefits, the re-weighting technique is still time-consuming, as it requires reading and processing a large data sample. On a $2.8 \mathrm{GHz} \mathrm{CPU}$, it takes more than an hour to re-weigh a 10-million-event sample. It is not practical to use this for every iteration of the fitting procedure. To solve this problem, we simplify the model used in fits to experimental data for the template morphing that we want to use in the fitting. We construct our function by calculating, through the re-weighting process, a histogram $H_{0}$ for the value of $\mathrm{R} \chi \mathrm{L}$ model parameters $x_{1 \ldots n}$. We then generate histograms $H_{1 \ldots n}$ corresponding to the case when one fit parameter $x_{1 \ldots n}$ is changed by $\Delta P_{1 \ldots n}$ to obtain an approximation of the first-order partial-derivative of this parameter. Leaving only the linear terms from the Taylor expansion of our fit function, the simplified fit function can be expressed as:

$$
F_{f i t}\left(P_{1}, P_{2}, P_{3}, \ldots, P_{n}\right)=H_{0}+\sum_{i}\left(P_{i}-x_{i}\right) \frac{H_{i}-H_{0}}{\Delta P_{i}} .
$$

where $P_{1 \ldots n}$ are the fit parameters. We then use algorithms from the MINUIT package [8], available through the ROOT framework [2], to fit this linear approximation at point $x_{1 \ldots n}$ of our model to the data. The second derivative (quadratic dependence) can be introduced as well:

$$
\begin{aligned}
F_{\text {fit }}^{(2)}\left(P_{1}, P_{2}, P_{3}, \ldots, P_{n}\right)=H_{0} & +\sum_{i}\left(P_{i}-x_{i}\right) \frac{H_{i}-H_{0}}{\Delta P_{i}}+ \\
& \frac{1}{2} \sum_{i j}\left(P_{i}-x_{i}\right)\left(P_{j}-x_{j}\right) \frac{H_{i j}-H_{i}-H_{j}+H_{0}}{\Delta P_{i} \Delta P_{j}} .
\end{aligned}
$$

Here, $H_{i j}$ means that both $i$-th and $j$-th parameter were shifted respectively by $\Delta P_{i}$ and $\Delta P_{j}$. If $i=j$, shift by $2 \Delta P_{i}$ is used. In practice, we were limiting our calculation to these diagonal terms only. Our function would then read:

$$
\begin{aligned}
\tilde{F}_{\text {fit }}^{(2)}\left(P_{1}, P_{2}, P_{3}, \ldots, P_{n}\right)=H_{0} & +\sum_{i}\left(P_{i}-x_{i}\right) \frac{H_{i}-H_{0}}{\Delta P_{i}}+ \\
& \frac{1}{2} \sum_{i}\left(P_{i}-x_{i}\right)^{2} \frac{H_{i i}-2 H_{i}+H_{0}}{\Delta P_{i}^{2}} .
\end{aligned}
$$

This stage requires a negligible amount of CPU time and results in the best possible values of the parameters for a linearized model created at point $P_{1 \ldots n}$. These values are used to calculate a new point in parameter space for which we repeat the above procedure. We iterate until the difference $P_{1 \ldots n}-x_{1 \ldots n}$ is sufficiently close to 0 , indicating convergence to a minimum.

With the further improvements described in Section 2.4, this approach yields relatively good preliminary results in just a few steps (10 to 20). However, this method is very slow. In order to reduce the statistical error form, the MC sample we need to use 
a big $\mathrm{MC}$ generated sample (relative to the measured data). If we require uncertainties of the MC-generated data to be 3 times smaller than those of the experimental data, then we need to use an MC sample 10 times larger than the experimental one. This means 20 million events (taking roughly 3.5 hours) for one iteration of the re-weighting process $^{4}$.

\subsection{Calculating the energy-dependent width of the $a_{1}$ resonance}

The following mechanisms of 3 -pion production are described by the $\mathrm{R} \chi \mathrm{L}$ model ${ }^{5}$ :

I. double resonance production: $\tau^{-} \rightarrow a_{1}^{-} \nu_{\tau} \rightarrow(\rho ; \sigma) \pi^{-} \nu_{\tau}$,

II. single resonance production: $\tau^{-} \rightarrow(\rho ; \sigma) \pi^{-} \nu_{\tau}$,

III. a chiral contribution (direct decay, without production of any intermediate resonances)

The main contribution to the width comes from the first mechanism ${ }^{6}$. This brings an additional complication for the fit because one needs to compute additionally the width function of the $a_{1}$ resonance that mediates the decay process. This function is a two-dimensional integral that needs to be calculated for every point in decay phasespace in each event in the MC sample. If it were, in fact, calculated every single time, it would degrade the performance of the $\mathrm{MC}$ generator by a factor of a thousand (taking into account all decays rejected in the MC process) To avoid this, TAUOLA uses a 1000-point pre-calculated table of the width of the $a_{1}$ resonance, which is later interpolated to obtain a precise value for each point in phase-space.

In the $\mathrm{R} \chi \mathrm{L}$ model, this table depends on several of the $\mathrm{R} \chi \mathrm{L}$ parameters as, for example, the mass of the $a_{1}$ and $\rho$ resonances ${ }^{7}$. Whenever one of these parameters changes, this table has to be recalculated; however, it can be kept for the wholeevent sample. Nonetheless, it requires these lengthy calculations to be performed for each set of parameters. During our first trials, we attempted to approximate the $a_{1}$ contribution by ignoring the calculation of $a_{1}$ width and using just the results computed for the starting point. However, as $a_{1}$ is a part of the model itself, it quickly became obvious that without these calculations, our fitting algorithm is not stable and may not be able to converge to the minimum [14]. Therefore, we had to include the $a_{1}$ width recalculation in each step of the process.

In our first approach, we used a 16-point Gaussian quadrature with an adaptable number of divisions fulfilling the precision requirement to calculate the integral. This integration routine was nested three times, increasing the precision requirement for each inner integral. A non-parallelized version of this code could take between one and two hours to calculate the 1000-point table (depending on cpu speed), making it one

\footnotetext{
${ }^{4}$ In the case of formula 1 , one iteration requires $n+1$ re-weighting procedures $(n$ denoting the number of parameters in the fit) that can be performed simultaneously. Using formula 2 increases this number to $n^{2}+1$, whereas using formula 3 requires only $2 n+1$.

${ }^{5}$ This list includes the $\sigma$ resonance added later as an improvement to the model (see Section 3.1).

${ }^{6}$ The exact forms of the hadronic currents for all three mechanisms are written in [16].

${ }^{7}$ For a list of parameters, see Table 4 of [16].
} 
third of the whole time needed for one iteration. Later in this paper, we will address other methods of improving the speed of calculating $a_{1}$ propagator needed for fits using semi-analytical calculation of one-dimensional distributions (see Section 3.3).

\subsection{Problems with linearization}

In our study, from the fit sample defined in Section 2.2, we have constructed onedimensional histograms of the variables where the experimental data is available as unfolded histograms as well. Our predictions from the model, thanks to formula 1, feature linear dependence on the model parameters. Alternatively, bilinear dependence can be introduced with the help of formula 2 or, to save time, with the simplification presented in formula 3.

While using this method helped us quickly set up the fitting framework, the limitations soon became obvious. First, the linear approximation tends to be unstable close to the minimum. That is because near the minimum, the linearized model can cause larger changes of $\chi^{2}$ than the actual changes from the model (see Figure 3). Depending on the shape of the function, this may cause the method to indefinitely jump around the minimum, resulting in a lack of convergence.

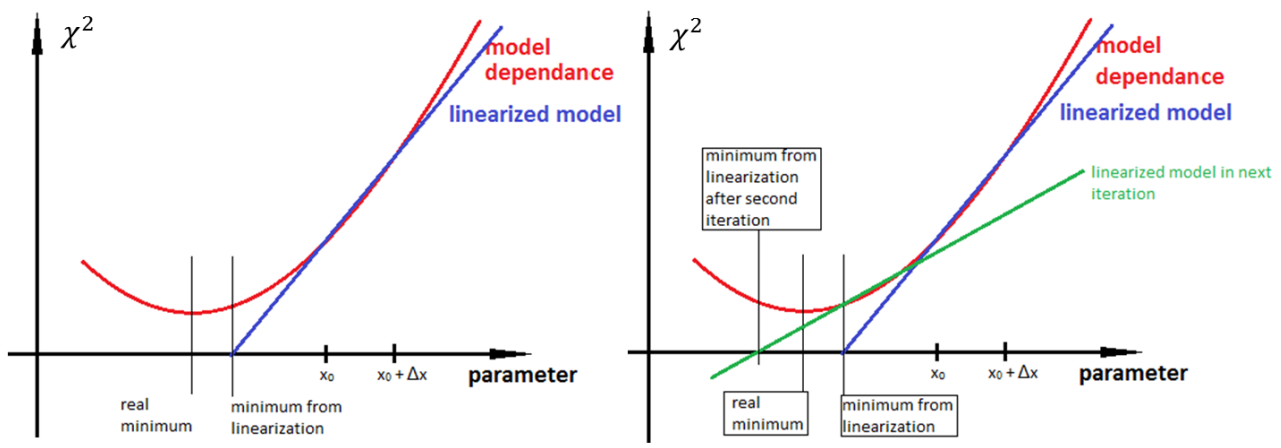

Figure 3. One-dimensional example of how linearization of parameter dependence of model affects fitting parameters to the data. Minimum of $\chi^{2}$ test between experimental data and model predictions is shifted from real minimum to minimum from linearization. In the second iteration (right-hand plot), the result will drastically change the first derivative for the third iteration.

This can be alleviated by calculating the result in one additional point for each parameter to calculate the estimation of the second derivative. Then, by requiring that the part dependent on the second derivative in the Taylor expansion is much smaller than linear terms ${ }^{8}$, we can estimate a region in which the linear approximation of

${ }^{8}$ The work on evaluating the stopping condition has not been completed. It will be a useful step in future work on this method. For the present study, a constant step size was used in this method to obtain general cross-check if the results and method described in Section 3 are not suffering from major technical or any other flaws. 
this parameter is valid ${ }^{9}$. Narrowing the range of the next step to this region helps stabilize the model, making it easy to get very good results with less iterations. On the other hand narrowing strongly the steps in parameter space close to the minimum, makes it extremely slow to converge. The second derivative can also be used to estimate the distance to the minimum and to define a stopping condition. Also, if additional samples are computed on separate cores, these calculations do not affect the computation time of a single iteration.

\subsection{Results and applications}

Development of this method was postponed to complete work on the semi-analytic method, which is described in the following sections. This decision was made to take advantage of the unfolded data from BaBar, which does not benefit from the increased versatility of the $\mathrm{C}$ template method. Because of this, further improvements were not added to this method.

Comparison of the best results obtained using this method (without the $\sigma$ resonance in the decay mechanisms, see the beginning of Section 2.3 and Section 3.1) to the data, can be seen in Figure 4. The current version of this method takes about two weeks to converge.
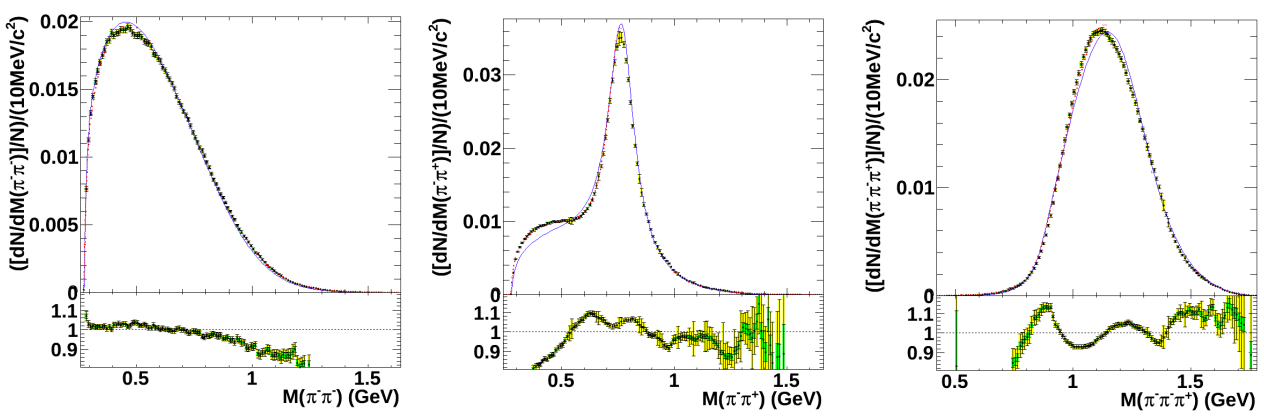

Figure 4. The results of the fits compared to the data. The differential decay width of the channel $\tau^{-} \rightarrow \pi^{-} \pi^{-} \pi^{+} \nu_{\tau}$ versus the invariant mass distributions is shown. BaBar experiment measurements (black points), results from the $\mathrm{R} \chi \mathrm{L}$ (blue line) and the CLEO tune (red dashed line) are overlaid. Ratio of new $\mathrm{R} \chi \mathrm{L}$ prediction to the data is at the bottom.

The goodness-of-fit for these results is $\chi^{2} / n d f=55264 / 401$.

This approach has proven to be invaluable in understanding the behavior of the fitted model and helped us estimate the potential of the model to represent the data. We have chosen to develop this method because of its vast applicability in different

${ }^{9}$ Alternatively, we could introduce an adaptive step size. Using first or second derivatives, we estimate the step size, fit the linear model to the data, and produce a new sample based on the results of the fits. We then compare the $\chi^{2}$ of this result to the $\chi^{2}$ of the linear model and adapt the step size based on the results. 
aspects of validating a physics model. For example, it can be used to fit data where experimental cuts have been applied. It can also be used with a re-weighting process, as described in Section 2.1, where each event is attributed with a weight dependent on the model used. This allows us to process an previously-generated event sample and analyze its characteristics using a different model.

These applications will definitely be useful in future analysis. However, to complete this analysis, we had to be able to thoroughly verify the model and the fitting framework itself. However, due to inefficiency of this method, some steps of the validation procedures could not be performed with satisfying results. For example, the random scan of parameter space mentioned in Section 4.3.2, which allows us to find a starting point closer to the minimum, significantly improved the efficiency of the fitting algorithm. To obtain more-precise results and to be able to validate them, a new approach had to be taken. This required changes both to the fitting framework and to the physics model itself.

Let us stress that at present, we use the gradient method mainly as a cross-check for the semi-analytical method described in Section 3, as this is our default method for the numerical results of the physics interest. This is why some of the aspects of the gradient method (if it was used to obtain physics results) are not fully explored. We will return to this aspect of our work in the future, once the gradient method will be central for the precision fitting.

\section{Semi-analytic method}

\subsection{Improving the physics model}

With the availability of unfolded data from the experiments, an alternative strategy using semi-analytic methods was developed. A set of semi-analytic distributions ${ }^{10}$ had been prepared that correspond to the data distributions available to us. This required a substantial effort on the experimental side as well. Using these, we were able to make a direct comparison with the data. While these distributions cannot be used in the manner described in Section 2.5, they allowed for more efficient techniques due to the efforts mentioned above.

We have also included the final state Coulomb interactions. The estimated effect of this interaction on the results was relatively low; but for completeness, it had to be tested [10].

As mentioned in [15] and seen on Figure 4 (middle plot), the main concern in the case of the modeled process was the low-mass region, which both the old CLEO model and the new $\chi$ model have problems representing. Because our model was unable to describe that region, we added the low-mass scalar $\sigma$ resonance [12], which has been used in previous experiments to describe the low mass region, to our model. While the

\footnotetext{
${ }^{10}$ These distributions use analytical integration methods supported with numerical integration of at most two variables.
} 
$\sigma$ resonance is not well-defined by the theory, may not be a true resonance, and is not part of the $\mathrm{R} \chi \mathrm{L}$ scheme, it has been added in a way that does not conflict with the $\mathrm{R} \chi \mathrm{L}$ scheme (see [10], eq. (3)-(7)). The impact of the $\sigma$ resonance was tested in [10].

Implementing changes in the model (contribution of the $\sigma$ state) significantly improved the agreement with the data. A new comparison of our starting point to the data has been shown in Figure 5. One can see that the agreement of the starting point is already better than the best result of our previous approach, especially when analyzing the ratio of the new model to the data from the middle plot.
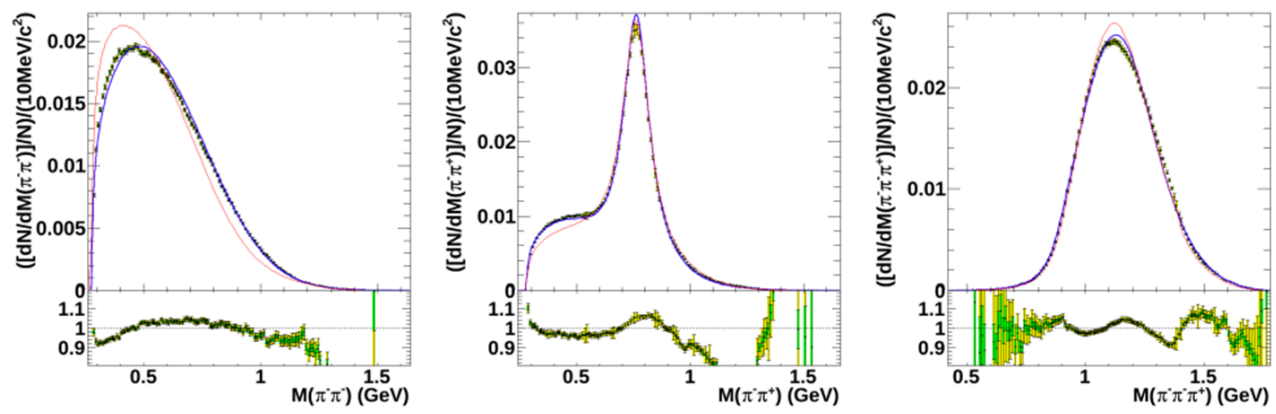

Figure 5. Starting point for the new fitting method, after applying improvements to the theoretical model. The goodness-of-fit for these results is $\chi^{2} / n d f=32413 / 401$. See caption of Figure 4 for description of the plots.

\subsection{Improving the fitting framework}

Parallel to the work on the physics model, the fitting framework has been improved as well. Switching from the MC simulation to semi-analytic distributions proved to be very effective. Just by using these functions instead of the full MC simulation we have gained more than a factor of 200. On a single core, without any optimizations, a single step took about one minute to compute ${ }^{11}$. And yet, there was still more room for improvement.

The new performance bottleneck was the three-dimensional integration. The previously-used 16-point Gaussian integration method was changed from a variablestep-size to a constant-step-size approach. Even though the first approach is more sophisticated, the second is better suited for fitting functions where even a small change of integrals can be significant. The fitting algorithm internally calculates the derivatives of the distributions with respect to all model parameters. The variablestep-size integration creates artificial discontinuities in the fitting function at points where step size changes. Such artifacts were problematic for the fitting procedure when near the minimum; therefore, we decided to use a more-stable method at a very small precision cost.

\footnotetext{
${ }^{11}$ At that moment, we were not taking into account the computation of the width of the resonance $a_{1}$.
} 
To further smoothen the integrand and improve convergence, a change of integration variables was introduced according to the following scheme:

$$
\begin{aligned}
& \int_{x_{1}}^{x_{2}} f(x) d x=\int_{0}^{1} g^{\prime}(t) f(g(t)) d t \\
& \text { where: } \quad y_{1}=\operatorname{arctg}\left(\frac{x_{1}-A^{2}}{A B}\right), \quad y_{2}=\operatorname{arctg}\left(\frac{x_{2}-A^{2}}{A B}\right) \text {, } \\
& x=g(t)=A^{2}+\operatorname{ABtg}\left(y_{1}+t\left(y_{2}-y_{1}\right)\right), \\
& g^{\prime}(t)=A^{2}+\frac{\left(g(t)-A^{2}\right)^{2}+A^{2} B^{2}}{A B} .
\end{aligned}
$$

This changes the integrated variable from $\mathrm{x}$ to $\mathrm{g}(\mathrm{t})$, introducing two adjustable parameters $(\mathrm{A}, \mathrm{B})$. This setup is commonly used to optimize the integration of functions describing resonances in particle physics, where parameter $\mathrm{A}$ is the resonance peak and $\mathrm{B}$ is the resonance width. In our calculations, we have chosen parameter A close to peak of $\rho$ resonance [12] (which can be seen in Figure 5, middle plot) and decided that parameter B should not be too narrow, to account for the width of the $a_{1}$ resonance (seen in Figure 5, right-hand plot). Based on the results from our benchmark tests, we have chosen $A=0.77, B=1.8$ without further consideration of possibly more optimal choices.

The change of variables and stability of semi-analytical functions allowed us to reduce the number of steps of the integration algorithm. While the variable-step-size integration could take from 6 to 18 steps for the selected integration domain, we have benchmarked several choices of constant-step-size approach.It turned out that the precision of calculations did not improve past the 3 steps. We decided to use 2 steps, as the difference between 2 and 3 was not significant. Applying these changes brought a gain of speed close to a factor of ten.

\subsection{Parallelization}

Building our fitting environment around the semi-analytical distributions allowed for an easy way to introduce parallelization. At this point, the only time-consuming operation was 3-dimensional integration. This job can easily be sub-divided into as many parts as needed, and each task can be computed independently. This makes parallelization straightforward; the only restriction in this manner is the technology used for this purpose.

Since part of the tests will have to be done by our collaborator on a computing cluster with unknown support for parallel computing, we had to prepare a method that would be as portable as possible. Fortunately, the Inter-Process Communication (IPC) methods of UNIX systems proved to be more than enough for this task. Therefore, we decided to base our algorithm on message queues.

This choice allows one to easily create an asynchronous communication link between the master program and any number of computing nodes without the need of 
any other IPC methods ${ }^{12}$ (see Figure 6 ). Since the operating system takes care of cashing sent messages and distributing them to the first node that is ready to receive the message, this simplifies the whole process down to few points that have to be taken into account when writing the master program and the computing nodes.

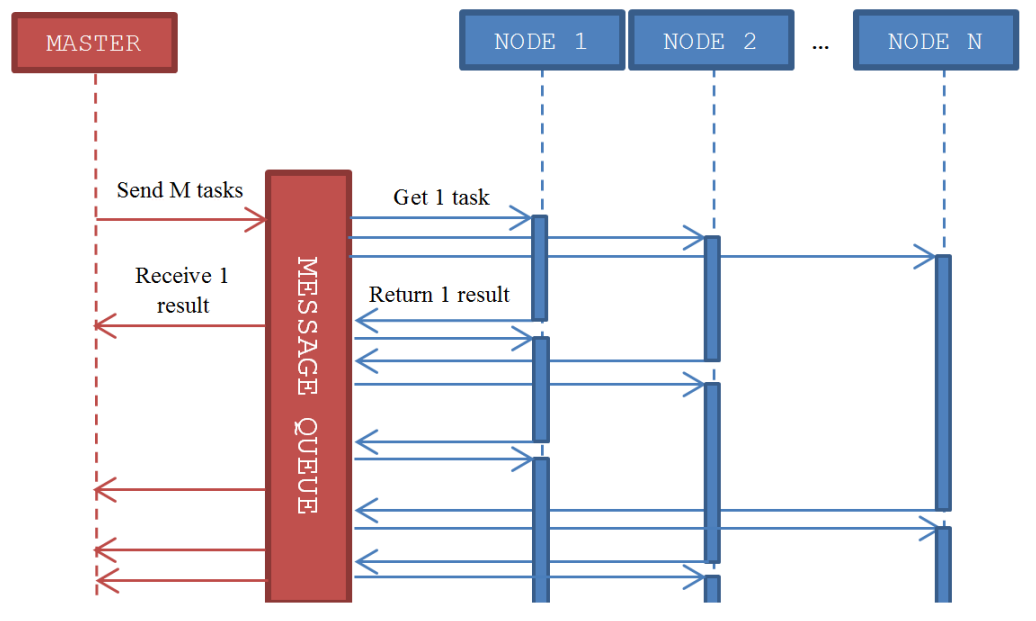

Figure 6. Diagram of communication between master program and computing nodes using message queue. Note that both master program and computing nodes can send and receive messages in an asynchronic way. Master program does not have to actively wait for nodes to finish their computations.

Our algorithms works as follows:

1. The master program divides the job into smaller tasks and sends a complete list of tasks to the queue, creating a pool of tasks for computing nodes. The ordering of the tasks is irrelevant.

2. Computing nodes wait for a message to appear in the queue, then proceed to compute the requested task and send the result back to the queue with proper ID for the master program to receive them.

3. The master program waits for a message with proper ID and gathers the results.

4. Computation ends when the number of messages received equals the number of messages sent.

This algorithm requires few things to take into consideration:

1. The master program must be prepared to receive data in any order.

2. A node that failed mid-computation (for any reason) will not return any results. A time-out must be put in place to decide when the master program has to re-send tasks that have not been computed.

\footnotetext{
${ }^{12}$ In our case, all nodes are homogeneous, which makes this task even simpler.
} 
Both issues are easy to resolve. With this algorithm in place, the number of computing nodes can be freely adjusted, allowing us to optimize the resources used versus computation time. Our tests showed a linear decrease in computation time with the increase of cores used for computation up to 24 cores. Above this threshold, communication becomes the main factor that slows down the progress. This could be mitigated by increasing the size of the tasks sent to one node, but we decided against further optimization as the computing time was satisfactory at this point.

\subsection{Approximating the width of the $a_{1}$ resonance}

As mentioned in Section 2.1, one of the key components of the model is the $a_{1}$ resonance width. Our first attempts used the same approximation as in the MC approach, where the width of the $a_{1}$ resonance is calculated only at the starting point of the fitting procedure. However, the lack of a proper recalculation of this resonance turned out to greatly influence the results. A fitting procedure that relies on $a_{1}$ width calculated only once ends in a minimum completely off the global minimum found when this width is properly recalculated for each point in parameter space.

Having wrong values of the $a_{1}$ width can skew the result, creating a minimum heavily correlated with a starting point for which the width was generated. This meant that we had to include the $a_{1}$ width in our calculations.

Since the calculation time of $a_{1}$ width significantly dominates the whole computation time, we decided to introduce an old method in use since 1992 to optimize the fitting framework (used to approximate the $a_{1}$ width). This approximation, based on a general knowledge of the shape of $a_{1}$ width distribution, uses a piecewise function built upon sets of polynomials to interpolate the $a_{1}$ width. It is parameterized by calculating the width in as few as 8 points. This approximation has been tested to show that it introduces deviations from the precise calculations less than $7 \%$ for low-mass region and less than $1 \%$ for the most important region.

Later on, when the parallelized calculation had been introduced, we were able to easily incorporate the precise calculations into the project. These calculations, however, still took the majority of computation time, so we left an approximated function as an option that can be used to quickly obtain preliminary results, as it may be important in future applications.

\section{Results}

\subsection{Final results of the fits}

Figure 7 shows the result of the fits using the semi-analytical method described in Section 3 compared to the data and results of the CLEO parameterization used so far by the experiments. The goodness of the fit is quantified by $\chi^{2} / n d f=6658 / 401$. This is the first case when agreement for a non-trivial $\tau$ decay channel was obtained between the BaBar data and the theoretical model and the systematic errors could 
be addressed. These comparisons can serve as a starting point for future precision studies of $\tau$ decays [13].
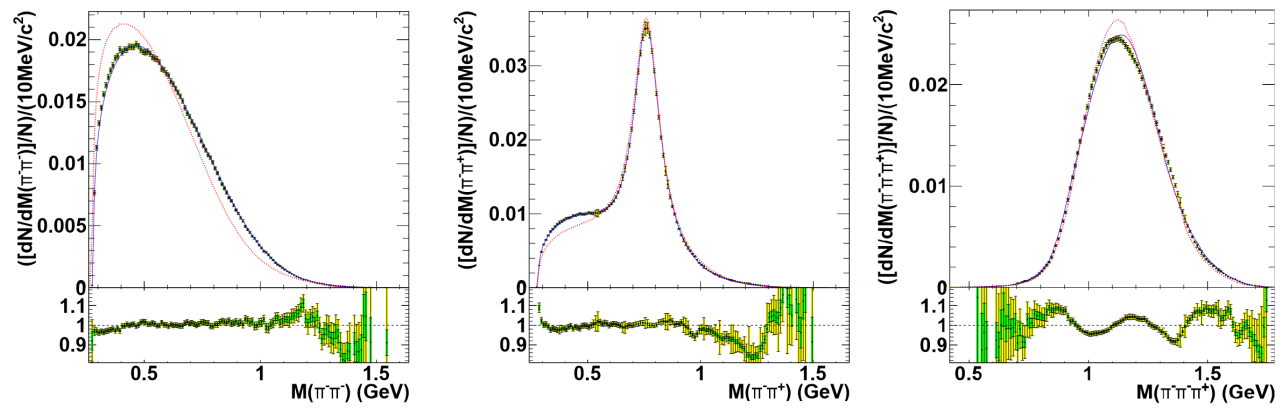

Figure 7. Final results of the fits. The goodness-of-fit for these results is $\chi^{2} / n d f=6658 / 401$. See caption of Figure 4 for description of the plots.

\section{Table 1}

Final results of the fits - parameter values and their ranges. The approximate uncertainty estimates from the MINUIT are 0.4 for $R_{\sigma}, 0.13$ for $M_{\rho^{\prime}}$ and below $10^{-2}$ for rest of the parameters.

\begin{tabular}{|c|c|c|c|c|c|c|c|c|c|c|c|c|c|c|c|}
\cline { 2 - 14 } \multicolumn{1}{c|}{} & $M_{\rho}$ & $M_{\rho^{\prime}}$ & $\Gamma_{\rho^{\prime}}$ & $M_{a_{1}}$ & $M_{\sigma}$ & $G_{\sigma}$ & $F$ & $F_{V}$ & $F_{A}$ & $\beta_{\rho}$ & $\alpha_{\sigma}$ & $\beta_{\sigma}$ & $\gamma_{\sigma}$ & $\delta_{\sigma}$ & $R_{\sigma}$ \\
\hline $\min$ & 0.767 & 1.350 & 0.300 & 0.990 & 0.400 & 0.400 & 0.088 & 0.110 & 0.100 & -0.370 & -10 & -10 & -10 & -10 & -10 \\
\hline $\max$ & 0.780 & 1.500 & 0.500 & 1.250 & 0.550 & 0.700 & 0.094 & 0.250 & 0.200 & -0.170 & 10 & 10 & 10 & 10 & 10 \\
\hline fit & 0.772 & 1.350 & 0.448 & 1.092 & 0.488 & 0.700 & 0.091 & 0.169 & 0.131 & -0.318 & -8.796 & 9.764 & 1.264 & 0.657 & 1.867 \\
\hline
\end{tabular}

Since the focus of this paper is on the technical aspects of this project, we refer the reader to [10] for physics-related results. Instead, we will focus on performance results and validation process of the fitting framework in this section.

\subsection{Performance}

Improvements to the fitting framework reduced computation time drastically. On a single $2.8 \mathrm{GHz}$ core with all approximations in place, a single iteration takes about one minute to complete. This computation time scales linearly with amount of cores used for computation, up to 24 cores; in this case, it takes about 3 seconds to compute one iteration.

As shown in Figure 8, 24 cores is the limit for which the time gain is the highest for a single master program. However, during performance tests, an algorithm for sending multiple tasks at once was used. A pre-calculated polynomial was used to estimate the time required for each task to complete, creating groups of tasks with balanced computation time. On 64 cores, such an algorithm took less than a second to 
complete a single iteration. Preliminary results were available in 2.5 hours. However, its use turned out to be impractical, as one 64-core job waited far longer in the computing cluster queue than three 24-core jobs.

The precise computation of the width of the $a_{1}$ resonance increases computation time by a factor of two. This reduces the $\chi^{2}$ only by about $10 \%$. In the final steps, we removed the approximation of using only the value at the bin center and replaced it with the correct treatment, where we integrate over the bin width. This makes the $\chi^{2}$ smaller by another $5-6 \%$ at a cost of 3 to 5 times slower computation. When both options are included and fits are performed from the point calculated without these improvements, the $\chi^{2}$ is reduced yet again by $5 \%$ to $6 \%$. Ultimately, the final result on 24 -core machine takes 2 to 3 days to complete, as compared to two weeks on a 32-core machine for a Monte-Carlo approach.

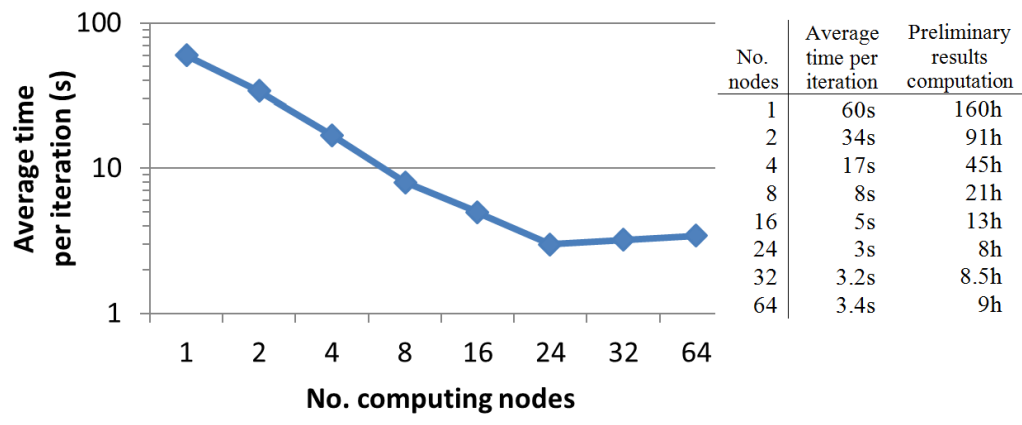

Figure 8. Scalability of the parallel distribution method using one message queue, one master program and sending one task per message. Above 24 cores, there is no improvement observed as the communication between computing nodes and master program starts domination over the computation time gain.

The ability to obtain preliminary results within one day and to produce precise results within three days gave us an opportunity to test a number of different fitting strategies and to thoroughly validate our approach. This also allowed us to perform advanced systematics studies in a reasonable time frame.

\subsection{Validating the results}

Having an efficient fitting framework at our disposal, we could dedicate more time to the validation process, which is a crucial step in the fitting process. Our validation process consisted of asserting statistical errors and correlation coefficients between the parameters, verifying that the result we have found is a global minimum and performing studies of the systematics errors.

\subsubsection{Statistical errors and correlations}

The analysis of statistical error and correlations between parameters was done using the HESSE algorithm from the MINUIT package, which simply calculates the full matrix 
of 2 nd derivatives and inverts it. The results show strong correlations between four parameters of the model indicating that the model has more free parameters than can be determined by the data. This is something that could have been expected and this issue was addressed in more details in [10].

\subsubsection{Convergence of the fitting procedure}

The purpose of the convergence test is to verify how the fitting procedure behaves when starting from different points. This test helps to verify that the minimum found by the fitting procedure is, in fact, a global minimum. It was done in the following steps:

- Random scan of parameters space. Since precision was of a lesser concern at this step, we have used all available approximations yielding a sample of 12,000 distinct parameter sets per hour using 240 cores.

- Having gathered more than 200k samples, we began searching for any patterns that could help us narrow down the region in parameter space where there is a larger chance of finding a global minimum.

- We choose a $1 \mathrm{k}$ sample of distributions for distinct parameter sets with the lowest $\chi^{2}$ and choose 20 points from this parameter sets in a way that maximized the distance between these points.

- We perform fits to the data using these 20 configurations as starting point for the independent fits.

The results have shown that more than $50 \%$ of the fits converge to the minimum shown as our final result. In other cases, either the minimization process fails due to the number of parameters being at their limit, or they converge to a local minima of distinguishably higher $\chi^{2}$ (by a factor of 10 or more). This indicates that our model has several local minima. At the same time, it points out that there is a good chance of our result being a global minimum.

\subsubsection{Systematic uncertainties}

We have estimated the systematic uncertainties using toy MC studies. Every toy MC sample has been generated under the Gaussian assumption using the Cholesky decomposition on systematic covariance matrix provided by the BaBar experiment to include the correlations between bins of the data histograms. The fit was re-run for one hundred samples to estimate the systematic uncertainties. It took less than a week using 320 cores to complete this step (which would be near to impossible to complete using MC approach).

\section{Summary and outlook}

We have presented a fitting strategy used for comparing $\mathrm{R} \chi \mathrm{L}$ currents for $\tau \rightarrow$ $\pi^{-} \pi^{-} \pi^{+} \nu_{\tau}$ with experimental data. The resulting improvements to the $\tau$ decays MC generator TAUOLA are installed in the LHC Computing Grid applications database [20]. 
We have presented two methods; one based on an MC simulation and the other based on semi-analytical distributions. While the first approach is significantly slower, it can be used in cases where analytic solutions are not available, such as presence of experimental cuts in data on multi-dimensional distributions.

We have presented improvements both to the theoretical model and to the fitting framework that significantly reduce computation time: from 2 weeks for the MCbased method to 2-3 days for method based on semi-analytic distributions. We have presented approximations introduced to the computations that allowed us to compute preliminary results within 8 hours. We have also introduced a scalable parallelization algorithm based on basic Inter-Process Communication methods of UNIX operating systems.

In the future, we are planning to use this framework to test the model against 2dimensional data for $\tau \rightarrow \pi^{-} \pi^{-} \pi^{+} \nu_{\tau}$ and for fits of $\tau \rightarrow K^{-} \pi^{-} K^{+} \nu_{\tau}$ decay channel. Fits for other decay modes will follow. As a final step of our work, we are planning to complete a model-independent fitting framework incorporating strategies presented in this paper with the ability to expand its application to multi-dimensional data and new fitting strategies.

\section{Acknowledgements}

We thank Ian M. Nugent for his collaboration on this project. Part of the computations were supported in part by PL-Grid Infrastructure (http://plgrid.pl/) and were performed on ACK Cyfronet computing cluster (http://www.cyfronet.krakow.pl/).

This project was partially financed from funds of Foundation of Polish Science, grant POMOST/2013-7/12 and funds of Polish National Science Centre under decisions DEC-2011/03/B/ST2/0010\%. This research was supported in part by the Research Executive Agency (REA) of the European Union under the rant Agreement PITNGA2012316704 (Higgs Tools). P.R. acknowledges funding from CONACYT and DGAPA through project PAPIIT IN106913.

\section{References}

[1] Actis S., et al.: Quest for precision in hadronic cross sections at low energy: Monte Carlo tools vs. experimental data. Eur. Phys. J., vol. C66, pp. 585-686, 2010. http://dx.doi.org/10.1140/epjc/s10052-010-1251-4.

[2] Antcheva I., Ballintijn M., Bellenot B., Biskup M., Brun R., et al.: ROOT: A C++ framework for petabyte data storage, statistical analysis and visualization. Comput. Phys. Commun., vol. 180, pp. 2499-2512, 2009. http://dx.doi.org/10.1016/j.cpc.2009.08.005.

[3] Asner D., et al.: Hadronic structure in the decay $\tau \rightarrow \pi^{-} \pi^{0} \pi^{0}$ and the sign of the tau-neutrino helicity. Phys. Rev., vol. D61, p. 012002, 2000. http://dx.doi.org/10.1103/PhysRevD.61.012002.

[4] Aubert B., et al.: The BaBar detector. Nucl. Instrum. Meth., vol. A479, pp. 1-116, 2002. http://dx.doi.org/10.1016/S0168-9002(01)02012-5. 
[5] Banerjee S., Kalinowski J., Kotlarski W., Przedzinski T., Was Z.: Ascertaining the spin for new resonances decaying into tau + tau- at Hadron Colliders. Eur. Phys. J., vol. C73, p. 2313, 2013. http: //dx.doi.org/10.1140/epjc/s10052-013-2313-1.

[6] Ecker G., Gasser J., Pich A., de Rafael E.: The Role of Resonances in Chiral Perturbation Theory. Nucl. Phys., vol. B321, p. 311, 1989. http://dx.doi.org/10.1016/0550-3213(89)90346-5.

[7] Jadach S., Kuhn J.H., Was Z.: TAUOLA: A Library of Monte Carlo programs to simulate decays of polarized tau leptons. Comput. Phys. Commun., vol. 64, pp. 275-299, 1990. http://dx.doi.org/10.1016/0010-4655(91)90038-M.

[8] James F., Roos M.: Minuit: A System for Function Minimization and Analysis of the Parameter Errors and Correlations. Comput. Phys. Commun., vol. 10, pp. 343-367, 1975. http://dx.doi.org/10.1016/0010-4655(75)90039-9.

[9] Kubota Y., et al.: The CLEO-II detector. Nucl. Instrum. Meth., vol. A320, pp. 66-113, 1992. http://dx.doi.org/10.1016/0168-9002(92)90770-5.

[10] Nugent I., Przedzinski T., Roig P., Shekhovtsova O., Was Z.: Resonance chiral Lagrangian currents and experimental data for $\tau^{-} \rightarrow \pi^{-} \pi^{-} \pi^{+} \nu_{\tau}$. Phys. Rev., vol. D88(9), p. 093012, 2013. http://dx.doi.org/10.1103/PhysRevD.88.093012.

[11] Nugent I. M. [BaBar Collaboration]: Invariant mass spectra of $\tau^{-} \rightarrow h^{-} h^{-} h^{+} \nu_{\tau}$ decays, Nucl. Phys. Proc. Suppl. 253-255, 38 (2014) [arXiv:1301.7105 [hep-ex]].

[12] Olive K., et al.: Review of Particle Physics. Chin. Phys., vol. C38, p. 090001, 2014. http://dx.doi.org/10.1088/1674-1137/38/9/090001.

[13] Pich A.: Precision Tau Physics. Prog. Part. Nucl. Phys., vol. 75, pp. 41-85, 2014. http://dx.doi.org/10.1016/j.ppnp.2013.11.002.

[14] Przedzinski T.: Test of influence of retabulation method on fitting convergence, 2011.

[15] Shekhovtsova O., Nugent I., Przedzinski T., Roig P., Was Z.: RChL currents in Tauola: implementation and fit parameters. Nucl. Phys. Proc. Suppl., 253-255, pp. 73-76, 2014.

UAB-FT-727 http://dx.doi .org/10.1016/j.nuclphysbps. 2014.09.018

[16] Shekhovtsova O., Przedzinski T., Roig P., Was Z.: Resonance chiral Lagrangian currents and $\tau$ decay Monte Carlo. Phys. Rev., vol. D86, p. 113008, 2012. http://dx.doi.org/10.1103/PhysRevD.86.113008.

[17] 't Hooft G.: A Planar Diagram Theory for Strong Interactions. Nucl. Phys., vol. B72, p. 461, 1974. http://dx.doi.org/10.1016/0550-3213(74)90154-0.

[18] Verkerke W., Kirkby D.P.: The RooFit toolkit for data modeling. eConf, vol. C0303241, p. MOLT007, 2003,

[19] Witten E.: Baryons in the 1/n Expansion. Nucl. Phys., vol. B160, p. 57, 1979. http://dx.doi.org/10.1016/0550-3213(79)90232-3.

[20] World LHC Computing Grid. 


\section{Affiliations}

\section{Tomasz Przedziński}

The Faculty of Physics, Astronomy and Applied Computer Science, Jagellonian University, Krakow, Poland, tomasz.przedzinski@cern.ch

\section{Pablo Roig}

Departamento de Física, Centro de Investigacion y de Estudios Avanzados del Instituto Politécnico Nacional, Apartado Postal 14-740, 07000 México D. F. 01000, México

\section{Olga Shekhovtsova}

Kharkov Institute of Physics and Technology 61108, Akademicheskaya,1, Kharkov, Ukraine. Institute of Nuclear Physics, PAN, Krakow, Poland

\section{Zbigniew Wąs}

Institute of Nuclear Physics, PAN, Krakow, Poland

\section{Jakub Zaremba}

Institute of Nuclear Physics, PAN, Krakow, Poland

Received: 4.07.2014

Revised: 30.10.2014

Accepted: 2.11.2014 